\title{
Extremely Rare Cardiac Involvement: Recurrent Pericardial Hydatid Cyst
}

\author{
Ibrahim Akpinar ${ }^{1}$, Sebahat Tekeli ${ }^{2}$, Taner Sen ${ }^{2}$, Nihat $\mathrm{Sen}^{2}$, Nurcan Basar ${ }^{2}$, \\ Kumral Ergun $\mathrm{Cagli}^{2}$, Serkan $\mathrm{Cay}^{2}$, Omac Tufekcioglu ${ }^{2}$ and Nilgun Isiksalan Ozbulbul ${ }^{3}$
}

\begin{abstract}
Echinococcus granulosus is a common infestation in sheep and cattle raising countries. Although it is typically encountered in liver and lung, rare cardiac involvement of this infestation has very important clinical complications such as heart failure, valve regurgitation, pericardial effusion-tamponade and arrhythmia. In addition, pericardial infestation is an extremely rare condition of Echinococcus granulosus. Here, we report a case of recurrent pericardial hydatid cyst presenting exertional dyspnea, palpitation and presyncope attacks in a 72-year-old man. The diagnosis of recurrent pericardial hydatid cyst was made by transthoracic echocardiography, computed tomography and surgical history
\end{abstract}

Key words: recurrent, pericardial, hydatid cyst

(Intern Med 51: 391-393, 2012)

(DOI: 10.2169/internalmedicine.51.6370)

\section{Case Report}

A 72-year-old man was referred to our cardiology department because of exertional dyspnea, palpitation and presyncope attacks lasting for a period of 6 months. He had a history of cardiac hydatid cyst (HC) operation due to pericardial involvement in 2004. Electrocardiogram revealed atrial fibrillation. His heart rate was 41 beats per minute. Transthoracic echocardiography displayed an ovoid shaped cystic mass (Fig. 1) which was $3.7 \times 2.5 \mathrm{~cm}$ in size and located in the pericardium of the left ventricular antero-lateral wall. The content of the cyst demonstrated increased echogenicity and a typical double-layered appearance with welldefined edges and internal trabeculations corresponding to daughter membranes. Thoraco-abdominal computed tomography (CT) revealed a hypodense and well-defined cystic mass lesion located in the posterior wall of the left ventricle and in the spleen. In a three-dimensional volumerendered reconstruction image of cardiac CT, the cystic mass was revealed to also extend from the lateral wall to the posterior wall of the left ventricle (Fig. 2, 3). Indirect hemagglutination assay (IHA; Cellognost- Echinococcosis,
Siemens Healthcare Diagnostics, Marburg, Germany) was strongly positive as 1:256 (Low positive titres of 1:32 to $1: 128$ and strongly positive titers above of $1: 128)$. Thoracoabdominal CT detected no mass related with primary tumour of the other organs. Diagnosis of recurrent pericardial HC was made according to clinical surgical history and imaging modalities supporting cystic echinococcosis.

There was no evident reason for the low heart rate. The localization of the cyst was not suitable to affect the conduction pathway. We considered that it could be related to sick sinus syndrome or that it was merely a coincidence. A permanent pacemaker was implanted due to symptomatic bradycardia. We suggested a cardiac surgery but the patient refused the re-operation. He was discharged with $15 \mathrm{mg} / \mathrm{kg}$ albendazole treatment twice in a day. After six-months of albendazole treatment, there was no change in echocardiographic findings.

\section{Discussion}

Cystic echinococcosis (CE) is caused by the larva of tapeworm Echinococcus granulosus existing as a complex of different strains. Recent study of CE has revealed that it is

${ }^{1}$ Department of Cardiology, Zonguldak Karaelmas University, Faculty of Medicine, Turkey, ${ }^{2}$ Department of Cardiology, Turkiye Yuksek Ihtisas Hospital, Turkey and ${ }^{3}$ Department of Radiology, Turkiye Yuksek Ihtisas Hospital, Turkey

Received for publication August 11, 2011; Accepted for publication November 3, 2011

Correspondence to Dr. Ibrahim Akpinar, dr.ibrahimakpinar@gmail.com 


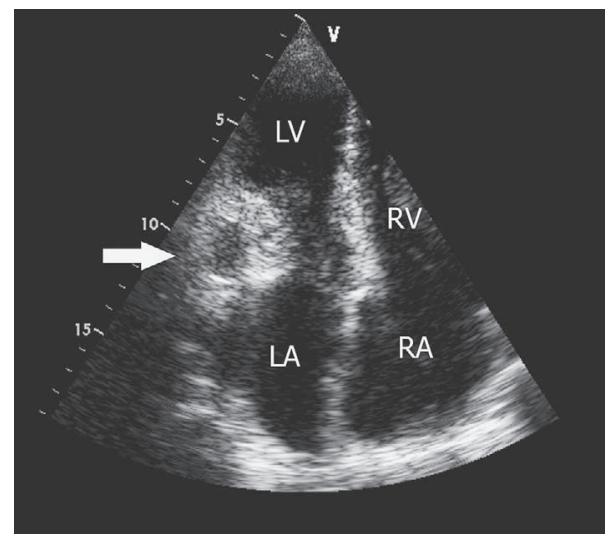

Figure 1. Transthoracic echocardiogram showing cystic mass adjacent to left ventricular anterolateral wall in apical four chamber view (arrow). LA: Left Atrium, LV: Left Ventricle, RA: Right Atrium, RV: Right Ventricle, HC: Hydatid Cyst

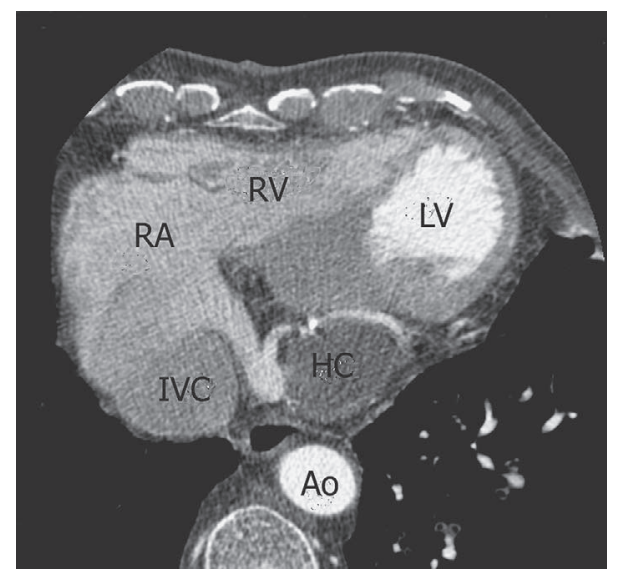

Figure 2. Computed tomography image compatible with hydatid cyst. LV: Left Ventricle, RA: Right Atrium, RV: Right Ventricle, IVC: Inferior Vena Cavae, Ao: Aorta, HC: Hydatid Cyst

not due to one species. To date, 10 distinct genotypes (G1G10) have been identified: 2 sheep strains (G1/G2), 2 bovid strains (G3 and G5), a horse strain (G4), a camel strain (G 6), 2 pig strains (G7 and G9) and 2 cervid strains (G8 and $\mathrm{G} 10)$. It means that $E$. granulosus is no longer a single species but rather a mixture of 5 species, E. granulosus sensu stricto (G1-G3), E. equinus (G4), E. oligarthrus (G5), E. canadensis (G6-G10), and E. felidis (1). Among these 5 species included in E. granulosus sensu lato, E. granulosus sensu stricto and E. canadensis are common in humans. However, we had no such molecular identification of the present case.

Cardiac involvement of hydatid cyst is a very uncommon form of E. granulosus infestation. Hydatid cysts of the heart are rarely detected and the prevalence is very low with a ratio of $0.5-2 \%$ of all cases (2). The mechanism of the larvae migration to the heart has several pathways such as coronary circulation, intestinal lymphatics, thoracic duct, the superior-

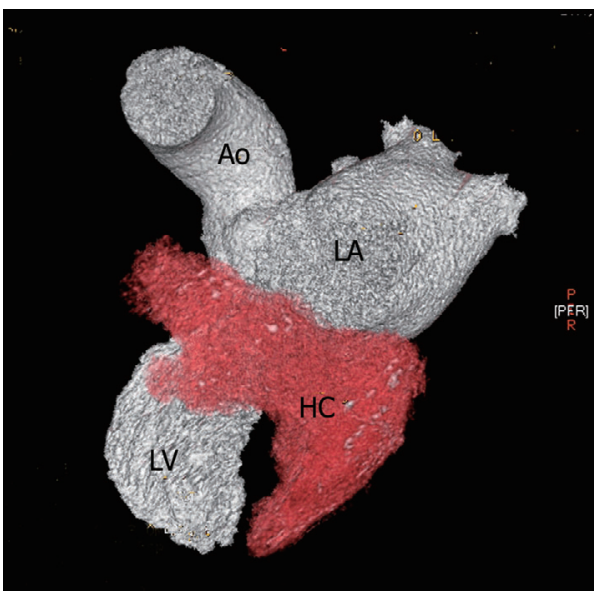

Figure 3. Three-dimensional volume rendered reconstruction image of computed tomography. LV: Left Ventricle, LA: Left Atrium, Ao: Aorta, HC: Hydatid Cyst

inferior vena cavae, hemorrhoidal veins and the pulmonary veins (3). The most frequent cardiac localization of a cyst is the left ventricle because of its thickness and rich blood supply (4).

In patients with an initial presentation of cardiac echinococcosis, transthoracic echocardiography is the first choice diagnostic modality, furthermore, computed tomography or magnetic resonance imaging is commonly used. In humans, the diagnosis is made by imaging techniques supported by serology. We applied the IHA test as a routine serologic test, although ELISA and immunoblot are more sensitive than IHA (5).

Typical appearances of $\mathrm{HC}$ in two-dimensional echocardiography, such as double-layered, well-defined edges and internal trabeculations, are very essential features. A diagnosis of recurrent pericardial $\mathrm{HC}$ was made according to cardiac surgical history related to $\mathrm{HC}$ and typical cystic mass existence.

Surgical intervention is the only definitive treatment and the results are satisfactory. Post-operative albendazole or mebendazole should be prescribed as an adjuvant therapy to prevent recurrence of the disease. If the lesions are heterogeneous, degenerative and have hyperechoic features which indicates inactive cysts, medical therapy can be discontinued. In the present case, recurrence may be the result of insufficient medical therapy. Sufficient data is not available on the mechanism of re-infestation after surgery. Recurrence of HC may also be related to small residual cysts. It is difficult to precisely distinguish these conditions because our patient did not come for echocardiographic follow-up after surgery.

Cystic echinococcosis has traditionally been most common in countries where large numbers of sheep and cattle are raised, however increased travel and immigration have made this condition a serious worldwide public health problem (6). Transthoracic echocardiography is not only the first choice diagnostic technique for cardiac $\mathrm{HC}$ but also valuable material to detect recurrence. Finally it should be performed for all patients who have a high suspicion index associated 
with this illness.

The authors state that they have no Conflict of Interest (COI).

\section{References}

1. Hüttner M, Nakao M, Wassermann T, et al. Genetic characterization and phylogenetic position of Echinococcus felidis (Cestoda: Taeniidae) from the African lion. Int J Parasitol 38: 861-868, 2008.

2. Dighiero J, Canabal EJ, Aguirre CV, Hazan J, Horjales JO. Echinococcus disease of the heart. Circulation 17: 127-132, 1958.
3. Kammoun S, Frikha I, Fourati K, et al. Hydatic cyst of the heart located in the interventricular septum. Can J Cardiol 16: 921-924, 2000.

4. Kaplan M, Demitras M, Cimen S, Ozler A. Cardiac hydatic cysts with intracavary expansion. Ann Thorac Surg 71: 1587-1590, 2001.

5. Aslan M, Yüksel P, Polat E, et al. The diagnostic value of Western blot method in patients with cystic echinococcosis. New Microbiol 34: 173-177, 2011.

6. Tufekcioglu O, Birincioglu CL, Arda K, Fansa I, Saritas A, Karahan M. Echocardiography findings in 16 cases of cardiac echinococcosis: proposal for a new classification system. J Am Soc Echocardiogr 20: 895-904, 2007.

(C) 2012 The Japanese Society of Internal Medicine http://www.naika.or.jp/imindex.html 